\title{
He and Ar beam scatterings from bare and defect induced graphite surfaces
}

\author{
Junepyo Oh, Takahiro Kondo*, Daigo Hatake, Yujiro Honma, Keitaro Arakawa, Takahiro \\ Machida and Junji Nakamura
}

Graduate School of Pure and Applied Sciences, University of Tsukuba, 1-1-1 Tennoudai, Tsukuba,

$$
\text { Ibaraki, Japan }
$$

*corresponding author: takahiro@ims.tsukuba.ac.jp

\begin{abstract}
The effect of the local electronic modification of the graphite surface on the gas-graphite interaction has been investigated by the molecular beam scattering technique. The angular intensity distributions of $\mathrm{He}$ and $\mathrm{Ar}$ beams scattered from pristine and defect induced graphite surfaces have been measured at various surface temperatures. From the He scattering results, the cross-section for the He diffuse scattering per defect is estimated as much as $113 \mathrm{~nm}^{2}$. The origin of the extremely large cross-section is ascribed to the modulated electronic states of graphite around the defect based on the STM measurements, which is due to the local breaking of the $\pi$ conjugated system of graphite. From the Ar scattering results, the effective mass of the graphite surface for the Ar collision has been estimated as $M=114 \mathrm{u}$ based on the analysis with the hard cube model. The new component appears in the scattering distribution of Ar for the defect induced graphite surface. The component has a larger peak position angle than that for the pristine graphite surface, indicating that the normal component of the translational energy of Ar atom was much lost by the collision at the electronically modified area of graphite.
\end{abstract}




\section{Keywords}

Highly oriented pyrolytic graphite (HOPG); Electronic states; Molecular beam; He scattering; Energy transfer;

\section{Introduction}

The gas-surface energy transfer process is closely related to the thermal properties of the material surface such as thermal conductivity and adiabaticity. It is also related to the surface chemical reactivity and catalytic activity because the gas-surface collision is the initial step of the surface chemical reactions. The graphite surface is known to have large anisotropy in the thermal properties between parallel and perpendicular to the c-axis of the crystal [1] because it is made from stacking of two-dimensional hexagonal lattices with weak interlayer interaction. When heavier gas such as Xe collides, the graphite surface behaves as "trampoline" due to a corporative motion of carbon atoms at the top-most graphene layer [2, 3]. The corporative motion is governed by the C-C bonds composed of $\sigma$-bonds and $\pi$ conjugated system of the graphite surface [4]. When the $\pi$ conjugated system is broken, the specific electronic states, "the non-bonding $\pi$ electronic states”, are known to form on the surface. The states are spontaneously formed near the zigzag edge of the graphite as 'edge-state' [5]. It has been found recently that the states are also formed around the vacancy defects on the graphite surface [6], i.e., the physical and chemical properties of the graphite surface around the defect are modified. It is thus expected that the collision dynamics of the molecule near the defects is different from that for the pristine graphite surface.

To understand the gas-surface energy transfer process at such sites of the graphite, molecular beam scattering techniques are available. It is expected that the He atom scattering can sensitively detect the electronically modified area of graphite quantitatively without any 
perturbation of the surface. The He atom scattering measurement is a widely used tool to analyze the surface structure because of its small mass and chemical inertness [7-15]. The sensitivity of the He for the detection of the morphological change is known to be extremely high owing to the large scattering cross-section of the low energy He atom towards adsorbed molecule or defect [7-15]. The objects on a surface cause the modulation of the attractive potential for He to the surface. On the other hand, the Ar scattering measurement can be used as a probe of the gas-surface energy transfer by a collision event. Because of the heavier mass of Ar than carbon of graphite, the scattering of Ar with thermal translational energy should dominantly show multi-phonon (and electron-hole pair excitation) inelastic scattering. Analyzing the mechanism of such an inelastic scattering event provides important insights such as effective mass of the surface, trapping probability, energy dissipation process of the projectile particle by the collision and so on. Manson has already established sophisticated analytical descriptions to represent such an inelastic scattering of the molecule by the classical scattering theory [16]. The theory is applicable for the wide scattering conditions even for the scattering of the molecule such as $\mathrm{CH}_{4}$ which has several internal energy modes. As another analytical theory, the hard cube model (HCM) [17] is well known as a classical binary collision theory to delineate trends in multi-phonon scattering of atoms and molecules from flat metal surfaces. The advantage of the model is its simplicity. It requires only one parameter of the effective surface mass to analytically calculate the multi-phonon scattering.

In this work, we have measured angular intensity distributions of He and Ar beams scattered from the pristine and defect induced $\left(\mathrm{Ar}^{+}\right.$ions sputtered) graphite surfaces at several surface temperatures. The modified electronic structure of the graphite surface has been discussed based on the He scattering results, scanning tunneling microscopy (STM) and spectroscopy (STS). The effects of the electronic modification upon the gas-surface collision 
dynamics of Ar are then studied with the analysis of HCM.

\section{Experimental}

The experimental apparatus used for the molecular beam scattering has already been described elsewhere $[18,19]$. The coherent length of the helium, the energy resolution and the angular spread of the beam in the apparatus were established as $\omega=16 \mathrm{~nm}, \Delta E / E=2.4 \%$, and $\Delta \theta=0.5^{\circ}$, respectively. The apparatus consists of five stainless-steel chambers. Each chamber is independently pumped to ultrahigh vacuum (UHV). A supersonic He (> $99.999 \%)$ or $\operatorname{Ar}(10 \%$ Ar seeded in $\mathrm{He}$ ) beam is generated by free-jet expansion from a pinhole of a cylindrical nozzle and skimmed using a conical skimmer. The diameter of the nozzle pinhole is set to $0.05 \mathrm{~mm}$. Stagnation pressure in the nozzle is controlled by a commercial gas regulator from 1 to $100 \mathrm{~atm}$. During the measurements, the temperature and stagnation pressure of the nozzle were kept at room temperature and $1.5 \mathrm{~atm}$, respectively. The translational energy $E_{i}$ and $\Delta E_{i} / E_{i}$ of the He or $\mathrm{Ar}$ beam at the same condition used in this work is experimentally identified by the time-of-flight technique as $E_{i}=64 \mathrm{meV}$ and $\Delta E_{i} / E_{i}=27.1 \%$ for He [4] and $E_{i}=314 \mathrm{meV}$ and $\Delta E_{i} / E_{i}=17.7 \%$ for $\operatorname{Ar}$ [18], respectively. STM images and STS spectra were taken at the different UHV low-temperature STM chamber [20] (UNISOKU, USM-1500) which has a base pressure of $2 \times 10^{-10}$ Torr.

The sample surface of highly oriented pyrolytic graphite (HOPG, ZYA-grade, Panasonic, $12 \mathrm{~mm} \times 12 \mathrm{~mm} \times 1.5 \mathrm{~mm}$ ) was cleaved in air by adhesive tape and then put into a UHV chamber. The HOPG sample was mounted to a sample holder which can be cooled down to 90 K by a cryogenic refrigerator head (Iwatani CryoMini S050) and can be heated by the infrared radiation from a hot $\mathrm{W}$ filament placed close to the back side of the HOPG sample. The surface temperature of HOPG was measured by a type-K thermocouple attached on the edge of the 
sample surface by a Ta clamp. Prior to the experiment, the HOPG sample was annealed at $900 \mathrm{~K}$ in UHV for 5 min in order to clean the surface. Defects of graphite were created by $\mathrm{Ar}^{+}$ion bombardment of HOPG at room temperature by using the commercial ion-gun (ANELVA, 5-KV ion gun). The sample was bombarded with $\mathrm{Ar}^{+}$ions of $300 \mathrm{eV}$ at the normal incidence. The total ion doses on the sample were set to be lower than $1.0 \times 10^{13} \mathrm{ions} / \mathrm{cm}^{2}$, which has been measured independently by the Faraday-cup.

After the defects creation, the sample was heated to $800 \mathrm{~K}$ to remove the implanted Ar. The surface morphology and electronic structure ware examined by low temperature STM with a PtIr tip at 5 K. STM images were recorded in constant-current mode.

The angular intensity distribution measurement is carried out for scattered He or Ar atoms from the surface by rotating the sample along the axis perpendicular to the beam line with an accuracy of $\pm 0.1^{\circ}$. Throughout the present study, both incident and scattering angles are defined with respect to the surface normal direction. The sum of the incident and scattering angles has been fixed at $90^{\circ}$.

\section{Result and Discussions}

In this section, the results of STM and STS were presented at first in section 3.1 as the characterization of the local geometric and electronic structures of the HOPG surface after the irradiation of $\mathrm{Ar}^{+}$ions. The angular intensity distributions of He and $\mathrm{Ar}$ from pristine and defect induced graphite surfaces are then presented with the analysis in section 3.2 and 3.3, respectively.

\subsection{STM and STS of defect induced graphite surface}

The typical STM image of the graphite surface after the irradiation of $\mathrm{Ar}^{+}$ions with 300 
$\mathrm{eV}$ is shown in figure 1a. The distribution of ion-induced defects estimated from the STM image is shown in figures $1 \mathrm{~b}$, where bright or dark spots with the width of 1-5 nm were observed. The atomic scale STM image of the HOPG surface with a defect is shown in Fig. 1c. It is well known that the $\beta$-carbon atoms of the graphite are basically imaged as brighter spots in the STM image of graphite [21]. Around the defect, there are two characteristic features: (i) Anisotropic propagation of an asteroid-like pattern with a 3 -fold symmetry; (ii) The $(\sqrt{3} \times \sqrt{3})$ R30 superstructure around the asteroid pattern. The asteroid pattern and superstructure have been reported previously as an image around a point defect of the graphite surface and they have been attributed to the electronic modification rather than the real atom arrangement $[22,23]$. We have measured STS spectra at different points in the vicinity of the point defect. The right-hand side

of Fig. 1c shows the spectra taken at the corresponding points A and B in Fig. 1c. Spectrum A shows a parabolic shape corresponding to the typical tails of the $\pi$ and $\pi^{*}$ states of graphite, suggesting that the defect does not significantly perturb the electronic structure at point A. On the other hand, a huge $d I / d V$ peak appears at $\approx 150 \mathrm{mV}$ in the spectrum of carbon at point $\mathrm{B}$, indicating that a new electronic state appears at the unoccupied region due to the perturbation of the electronic states of graphite by the defect. We have assigned the states as the non-bonding $\pi$ electronic states of graphite. They propagate non-uniformly up to 3-4 nm from the defect. The details of the states assignment, states propagation, states energy and states intensity are discussed in detail elsewhere [6].

\subsection{Angular intensity distribution of He}

The angular intensity distributions of He scattered from the sample surface are shown in figure 2. The distribution from the pristine HOPG surface significantly depends on the surface temperature. The dependence shows the transition of the scattering event from quantum 
mechanical scattering to the classical mechanical scattering of He as has been reported in our previous work [4]. At $150 \mathrm{~K}$, the elastic scattering component at $\theta_{\mathrm{f}}=45^{\circ}$, i.e., specularly reflected intensity, was clearly identified as a sharp peak. Two diffraction peaks at around $35^{\circ}$ and $55^{\circ}$ were also identified as the first order diffraction peaks of $(1,0)$ and $(-1,0)$ as shown by arrows. The result suggests that the pristine HOPG surface has large-size domains, each of which has a uniform lattice arrangement of graphite. With the increase of the surface temperature, the elastic scattering component decreases due to the Debye-Waller effect $[11,12]$. At the higher surface temperatures such as at $400 \mathrm{~K}$, no sharp elastic peak could be observed at $45^{\circ}$ in the distribution, suggesting that the scattering event purely occurs by the classical mechanical scattering.

Figure $2 \mathrm{~b}$ shows the results for the HOPG surface after the $\mathrm{Ar}^{+}$ions sputtering. The specularly reflected He intensity (intensity at $45^{\circ}$ ) was remarkably lower than that for the pristine HOPG surface, particularly at $150 \mathrm{~K}$. The decrease is originated from the formation of defects on the terrace of graphite by the $\mathrm{Ar}^{+}$ions sputtering. It is well known that the scattering of the thermal energy He atoms from the solid surface is extremely sensitive to the surface structure. The He atom shows diffuse scattering not only at defects (such as vacancy, step, kink, adatoms and specifically charge modulated site) but also at flat terrace area of the surface around the defect due to the change in the attractive potential for the incoming He to the surface. Such a diffuse scattering component has little contribution to the specularly reflected intensity $[9,10,24]$. With the increase of the surface temperature, the specularly reflected intensity at 150 $\mathrm{K}$ significantly decreased due to the Debye-Waller effect. At $400 \mathrm{~K}$, it became almost impossible to distinguish the difference of the angular intensity distribution between the pristine and the $\mathrm{Ar}^{+}$sputtered HOPG surfaces.

The decay of the specularly reflected intensity of He due to the presence of the defects 
can be quantified by using the concept of the cross-section for diffuse scattering. The cross-section of He atom scattering is known to be large, e.g., that for the CO/Pt(111) surface at very low CO coverage $\left(\theta_{\mathrm{CO}} \leqslant 0.01\right)$ is about $1 \mathrm{~nm}^{2}[9,10]$. In order to obtain the cross-section for the He diffuse scattering per defect on graphite, we have used the following simple equation [24];

$$
1-\frac{I}{I_{0}}=n_{S} \Sigma \Theta
$$

where, $I_{0}$ and $I$ are specularly reflected intensity of He from the pristine HOPG and the $\mathrm{Ar}^{+}$ sputtered HOPG, respectively, $n_{S}$ is the number of graphite atoms per unit area $\left(4 \times 10^{19}\right.$ atoms $\left./ \mathrm{m}^{2}\right), \Sigma$ is the cross-section for the He diffuse scattering, and $\Theta$ is the coverage of defects. The coverage is defined as the ratio of the number of defects to that of surface carbon atoms. The coverage of the defects was estimated as 0.0017 based on several STM images such as that shown in figure 1a [25]. The cross-section for the He diffuse scattering $(\Sigma)$ derived from the results at $150 \mathrm{~K}$ is $113 \mathrm{~nm}^{2}$. It is two orders of magnitude larger value compared to the typical cross-sections for the He diffuse scattering by the adsorbates on metal surface. Even considering the average diameter of defects which has been estimated about $3 \mathrm{~nm}$ from STM (figure 1b), it is still one-order of magnitude larger than typical value.

The origin of the large cross-section can be interpreted due to the long range electronic perturbation of the graphite surface around the defect. As shown in figure 1a, STM measurements have revealed that the $(\sqrt{3} \times \sqrt{3}) \mathrm{R} 30^{\circ}$ superstructure appears around the defect of the graphite surface. The structure has been ascribed to the charge density oscillations similar to Friedel oscillations [22, 23]. Our STS measurements directly further indicate the change in the electronic structure around the defect as shown in figure. 1b. Such an electronically modulated area is probably regarded as the defect area for the He atom incoming to the graphite surface, i.e., He atom sensitively detects the electronically modulated area around the defect of the 
graphite surface. In other words, the graphite surface looks flat to the STM around the superstructure, but it is corrugated to the He beam (either in the attractive or repulsive part).

\subsection{Angular intensity distribution of Ar}

Figure 3a shows the angular intensity distributions of Ar scattered from the pristine HOPG surface at a wide surface temperature range. The distribution has a single peak at around $54^{\circ}$ and has a quite broader distribution width compare to the case of He. The peak positioned at a higher scattering angle than $45^{\circ}$ indicates that the normal component of the translation energy of the Ar atom was lost by the collision with the graphite surface. Based on the peak position angle, it can be roughly estimated [26] that the Ar atom transfers 33\% of the normal component of the translational energy to the surface by the single collision. With the increase of the surface temperature, the peak shifts towards lower scattering angles, suggesting that the energy loss of Ar by the collision becomes small. This can be interpreted by the increased motion of the surface atoms at higher surface temperatures, i.e., the velocity of the surface carbon atoms collide with the incoming Ar atom becomes large with the increase of the surface temperature. The calculated HCM distributions well reproduces the dependence of the surface temperature as shown by the peak position in figure 4, where the effective mass used for the HCM calculation was set at $M=114 \mathrm{u}$ which corresponds to the weight of nine and a half carbon atoms. The surface effective mass derived by fitting experimental data is often greater than the real mass in many theoretical model analyses [4, 18, 27-42], which is attributed to the corporative motion of the surface atoms [2]. In the case of He and Xe scattering from HOPG, the effective masses have been reported as $M=72 \mathrm{u}$ [4] and $M=310 \mathrm{u}$ [41], respectively. The derived effective mass $M=$ $114 \mathrm{u}$ for the Ar scattering is heavier than the mass for He and lighter than the mass for Xe. The differences are probably caused by the difference of the size of the incoming atom to the HOPG 
surface; larger atom interacts with larger number of carbon atoms at the single collision event.

The angular intensity distributions of $\mathrm{Ar}$ from the $\mathrm{Ar}^{+}$sputtered HOPG surface are shown in figure $3 \mathrm{~b}$ as a dependence of the surface temperature. In every surface temperature, the peak position angles are located at a larger scattering angle compared to the case from the pristine HOPG surface as shown in figure 4. It means that the defects of graphite cause the larger energy loss of the Ar atom during the collision with the surface. In order to investigate the change of the energy transfer in detail, analytical fitting of the results for the $\mathrm{Ar}^{+}$sputtered HOPG surface are performed base on the results for the pristine HOPG surface as shown in figure 5. In the fitting analysis, the distribution obtained from the pristine HOPG surface (figure 3a), component 1 , and the additional component, component 2 , are used to precisely fit the results. It turned out that the component 2 is the distribution with the peak at the scattering angle of $64^{\circ}$. The component 2 should be related to the defects of the graphite surface because it does not appear for the scattering on the pristine HOPG surface. The ratio between components 2 and 1 is more than $10 \%$, which is larger than the total area of defects on the surface of $2 \%$ [43]. This suggests that the component 2 consists of the scattering of Ar not only from the defect itself but also probably from the electronically modified area around the defect of the graphite surface. The larger peak position angle of the component 2 indicates the larger energy loss of $\mathrm{Ar}$ by the collision with the electronically modified area of the graphite surface. Based on the peak position angle, it can be roughly estimated that the Ar atom transfers as much as $58 \%$ of the normal component of the translational energy to the surface by the single collision around defects site. The results presented here indicated a heterogeneous character in the energy transfer between graphite surface and molecules.

The graphite surface consists of the $\pi$ conjugated system which originates from the resonance of the $2 \mathrm{p}_{\mathrm{z}}$ electron orbital of the carbon. Therefore, the Ar atom may interact with the 
delocalized $\pi$ electron cloud of the pristine HOPG, as suggested by the large effective mass of 114 u. However, once C-C bonds of graphite are broken by the $\mathrm{Ar}^{+}$ions irradiation, the localized non-bonding $\pi$ electronic states are induced not only at defect but also at around the defect of the graphite surface as shown in figure 1 due to the breaking of the $\pi$ conjugated system [6]. Such a localized $\pi$ electron leads to the weak C-C binding energy. Indeed, low energy shift of the phonon modes has been reported at such an electronically modified carbon atoms around the Pt cluster on graphite based on the inelastic electron tunneling spectroscopy (IETS) [20]. Because of the weak C-C bonds of the graphite around the defect, the corporative motion of the carbon towards the colliding Ar may not exert effectively. The larger energy transfer of Ar to the graphite surface therefore occurs around the defect of the graphite surface.

\section{Conclusion}

We have investigated the effect of the local electronic modification of the graphite surface on the gas-graphite interaction. The angular intensity distributions of He and Ar beam scattered from the pristine and the defect induced HOPG surfaces have been measured at various surface temperatures. In the results of the He atom scattering, the specularly reflected intensity of He significantly decreases after inducing the defects by $\mathrm{Ar}^{+}$ions sputtering. The cross-section for the He diffuse scattering per defect has been estimated as $113 \mathrm{~nm}^{2}$. It is two orders of magnitude larger compared to the typical cross-sections for the He diffuse scattering by the adsorbates on metal surfaces. The origin of the large cross-section has been ascribed to the modulated electronic states of the graphite surface around the defect based on the measurements of STM and STS. The result indicates that the incident He atom has a high sensitivity to detect the change of electronic states of the graphite surface. Contrary to the He scattering, Ar dominantly shows inelastic scattering due to the larger mass than He. Based on 
the analysis of the scattering results of Ar from graphite with the hard cube model, the effective mass of the graphite surface for the Ar collision has been estimated as $M=114 \mathrm{u}$. In the case of Ar atom scattering, the peak angle of the distribution for the defect induced HOPG is shifted to a higher scattering angle by about $0.8^{\circ}$. The shift was caused by the appearance of a new distribution component for the defect induced HOPG surface. The component has a larger peak position angle than that for the pristine HOPG surface, indicating that the normal component of the translational energy of Ar atom was much lost by the collision at the electronically modified area of graphite.

\section{Acknowledgement}

We appreciate financial support from NEDO (New Energy and Industrial Technology Development) and by the Ministry of Education, Culture, Sports, Science and Technology (MEXT), through “Grant-in-Aid for Young Scientists (B) 21760023 


\section{References}

[1] Gurney R W 1952 Phys. Rev. 88 465; DeSorbo W and Tyler W W 1953 J. Chem. Phys. 21 1663.

[2] Grimmelmann E K, Tully J C and Cardillo M J 1980 J. Chem. Phys. 721039.

[3] Watanabe Y, Yamaguchi H, Hashinokuchi M, Sawabe K, Maruyama S, Matsumoto Y and Shobatake K 2005 Chem, Phys. Lett. 413331.

[4] Oh J, Kondo T, Hatake D and Nakamura J 2009 Surf. Sci. 603895.

[5] Stein S E and Brown R L 1987 J. Am. Chem. Soc. 109 3721; Kobayashi K 1993 Phys. Rev. B, 48 1757; Klein D J 1994 Chem. Phys. Lett. 217 261; Fujita M, Wakabayashi K, Nakada K and Kusakabe K 1996 J. Phys. Soc. Jpn. 65 1920; Nakada K, Fujita M, Dresselhaus G and Dresselhaus M S 1996 Phys. Rev. B 54 17954; Kawai T, Miyamoto Y, Sugino O and Koga Y 2000 Phys. Rev. B 62 R16349

[6] Kondo T, Honma Y, Oh J, Machida T and Nakamura J 2010 submitted

[7] Toennies J P 1993 J. Phys.: Condens. Matter. 5 A25.

[8] Heinz K, Müller K, Engel T and Rieder K H 1982 Structural Studies of Surfaces (Springer, Berlin).

[9] Poelsema B and Comsa G 1989 Scattering of Thermal Energy Atoms from Disordered Surfaces (Springer Tracts in Modern Physics, Springer-Verlag, Berlin)

[10] Scoles G Atomic and Molecular Beam Methods (Oxford University Press, New York, 1988), Vol. 1; (1992), Vol. 2.

[11] Kress W and Wette F W 1991 Surface Phonons (Springer, Berlin).

[12] Hofmann F and Toennies J P 1996 Chem. Rev. 961308.

[13] Farias D and Rieder K H 1998 Rep. Prog. Phys. 611575.

[14] Jardine A P, Ellis J and Allison W 2002 J. Phys.: Condens. Matter 146173. 
[15] Graham A P 2003 Surf. Sci. Rep. 49115.

[16] Manson J R 2008 “Energy Transfer to Phonons in Atom and Molecule Collisions with Surfaces", Handbook of Surface Science 3, 54; edited by E. Hasselbrink and B. I . Lundqvist.

[17] Logan R M and Stickney R E 1966 J. Chem. Phys. 44195

[18] Kondo T, Kato H S, Yamada T, Yamamoto S and Kawai M 2006 Eur. Phys. J. D 38129.

[19] Kondo T, Kato H S, Bonn M and Kawai M 2007 J. Chem. Phys. 127094703.

[20] Kondo T, Iwasaki Y, Honma Y, Takagi Y, Okada S, and Nakamura J, 2009 Phys. Rev. B 80 233408.

[21] Tománek, D, Steven G L, Jonathon M H, David W A, Thomson R E, Ganz E and Clarke J 1987 Phys. Rev. B 35 7790; $\beta$-carbon atoms are the carbon atoms located above the centers of the hexagons of the layer beneath; the remaining carbon atoms are $\alpha$-carbon atoms.

[22] Mizes H A and Foster J S 1989 Science 244559.

[23] Ruffieux, Melle-Franco M, Gröning O, Bielmann M, Zerbetto F, and Gröning P 2005 Phys. Rev. B 71153403.

[24] Poelsema B, Zwart S T and Comsa G 1982 Phys. Rev. Lett. 49578.

[25] The total area of the defect obtained from the result of STM image occupies about $4.7 \%$ of the surface area.

[26] We have calculated the energy transfer by assuming that only the normal component of the translational energy of Ar is transferred to the surface by the collision. In the calculation, we have taken into account of our experimental condition that the sum of the incident and scattering angles has been fixed at $90^{\circ}$.

[27] Moroz I and Manson J R 2004 Phys. Rev. B 69205406.

[28] Moroz I, Ambaya H and Manson J R 2004 J. Phys.: Condens. Matter 16 S2953.

[29] Moroz I and Manson J R 2005 Phys. Rev. B 71113405. 
[30] Kondo T, Tomii T and Yamamoto S 2006 Chem. Phys. 320140.

[31] Velic D and Levis R J 1997 Chem. Phys. Lett. 26959.

[32] Kondo T, Okada R, Mori D and Yamamoto S 2004 Surf. Sci. 566-568 1153.

[33] Kondo T, Mori D, Okada R and Yamamoto S, 2004 Jpn. J. Appl. Phys. 431104.

[34] Kondo T, Tomii T, Yagyu S and Yamamoto S 2001 J. Vac. Sci. Technol. A 192468.

[35] Armand G, Lapujoulade J and Lejay Y 1977 Surf. Sci. 63143.

[36] Arumainayagam C R, McMaster M C, Schoofs G R and Madix R J 1989 Surf. Sci. 222213.

[37] Wight A C and Miller R E 1998 J. Chem. Phys. 1091976.

[38] Tomii T, Kondo T, Hiraoka T, Ikeuchi T, Yagyu S and Yamamoto S 2000 J. Chem. Phys. 1129052.

[39] Kondo T, Tomii T, Hiraoka T, Ikeuchi T, Yagyu S and Yamamoto S 2000 J. Chem. Phys. 1129940.

[40] Berenbak B, Zboray S, Riedmuller B, Papageorgopoulos D C, Stolteb S and Kleyn A W 2002 Phys. Chem. Chem. Phys. 468.

[41] Watanabe Y, Yamaguchi H, Hashinokuchi M, Sawabe K, Maruyama S, Matsumoto Y and Shobatake K 2006 Euro. Phys. J. D 38103.

[42] Kondo T, Mori D, Okada R, Sasaki M and Yamamoto S 2005 J. Chem. Phys. 123114712.

[43] To estimate the coverage of the defect, total area of defects used for the Ar scattering experiment were calculated based on the change in the specularly reflected intensity of He, where the derived scattering cross section of $113 \mathrm{~nm}^{2}$ (see Sec. 3.2) was used for the calculation. In the sample used for Ar scattering measurements, the total area of ion-induced defects is about $2 \%$ of the surface area. We note here that when the defect density of the HOPG surface becomes larger, the component 2 in the Ar distribution disappears and broader distribution of component 1 is observed possibly due to the large amount of corrugation at the damaged surface. 


\section{Figure captions}

Figure 1

(a) Typical STM image of the HOPG surface after the irradiation with $\mathrm{Ar}^{+}$ions at $300 \mathrm{eV}$ $\left(100 \times 100 \mathrm{~nm}^{2}\right.$, sample bias $V_{\mathrm{s}}=300 \mathrm{mV}$ and tunneling current $\left.I_{\mathrm{t}}=100 \mathrm{pA}\right)$. (b) Height-width distribution of ion-induced defects. (c) Left; the STM image near the defect of the graphite surface $\left(10 \times 10 \mathrm{~nm}^{2}\right.$, sample bias $V_{\mathrm{s}}=300 \mathrm{mV}$ and tunneling current $\left.I_{\mathrm{t}}=152 \mathrm{pA}\right)$, right; the STS spectra taken at the points A and B of the image.

Figure 2

The surface temperature dependence of the angular intensity distribution of He scattered from (a) the pristine HOPG surface and (b) the $\mathrm{Ar}^{+}$ions sputtered HOPG surface. Incidence energy of He was set as $64 \mathrm{meV}$.

Figure 3

The surface temperature dependence of the angular intensity distribution of Ar scattered from (a) the pristine HOPG surface and (b) the $\mathrm{Ar}^{+}$ions sputtered HOPG surface at an incidence energy of $314 \mathrm{meV}$.

Figure 4

Peak position angle of the angular intensity distribution of Ar scattered from the pristine HOPG surface ( $\square$ ) and the $\operatorname{Ar}^{+}$ions sputtered HOPG surface $(\bullet)$ at various surface temperature. The dashed line indicates the results of hard-cube model calculation with effective mass of $114 \mathrm{u}$.

Figure 5 
The analytical fitting results of the angular intensity distribution of $\mathrm{Ar}$ scattered from the $\mathrm{Ar}^{+}$ions sputtered HOPG surface. The solid curve is an experimental data; blue dotted curve (component 1 ) is the same component with the angular intensity distribution of Ar scattered from the pristine HOPG surface (i.e. the results in Figure 3a), and red dotted curve (component 2) is a new component introduced to fit the result. 
Figure 1

Junepyo Oh, Takahiro Kondo, Daigo Hatake, Yujiro Honma, Keitaro Arakawa, Takahiro Machida and Junji Nakamura
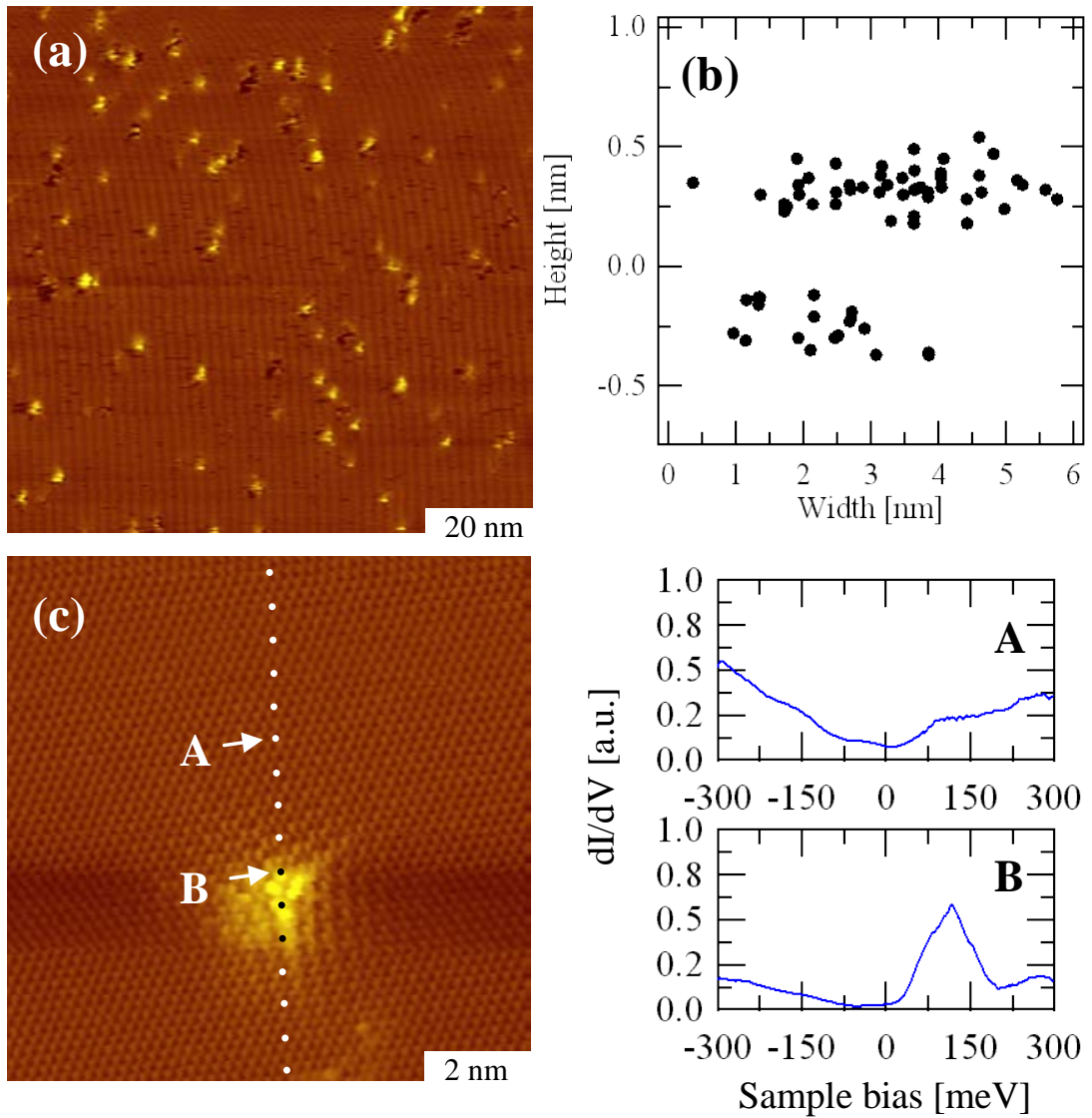
Figure 2

Junepyo Oh, Takahiro Kondo, Daigo Hatake, Yujiro Honma, Keitaro Arakawa, Takahiro Machida and Junji Nakamura
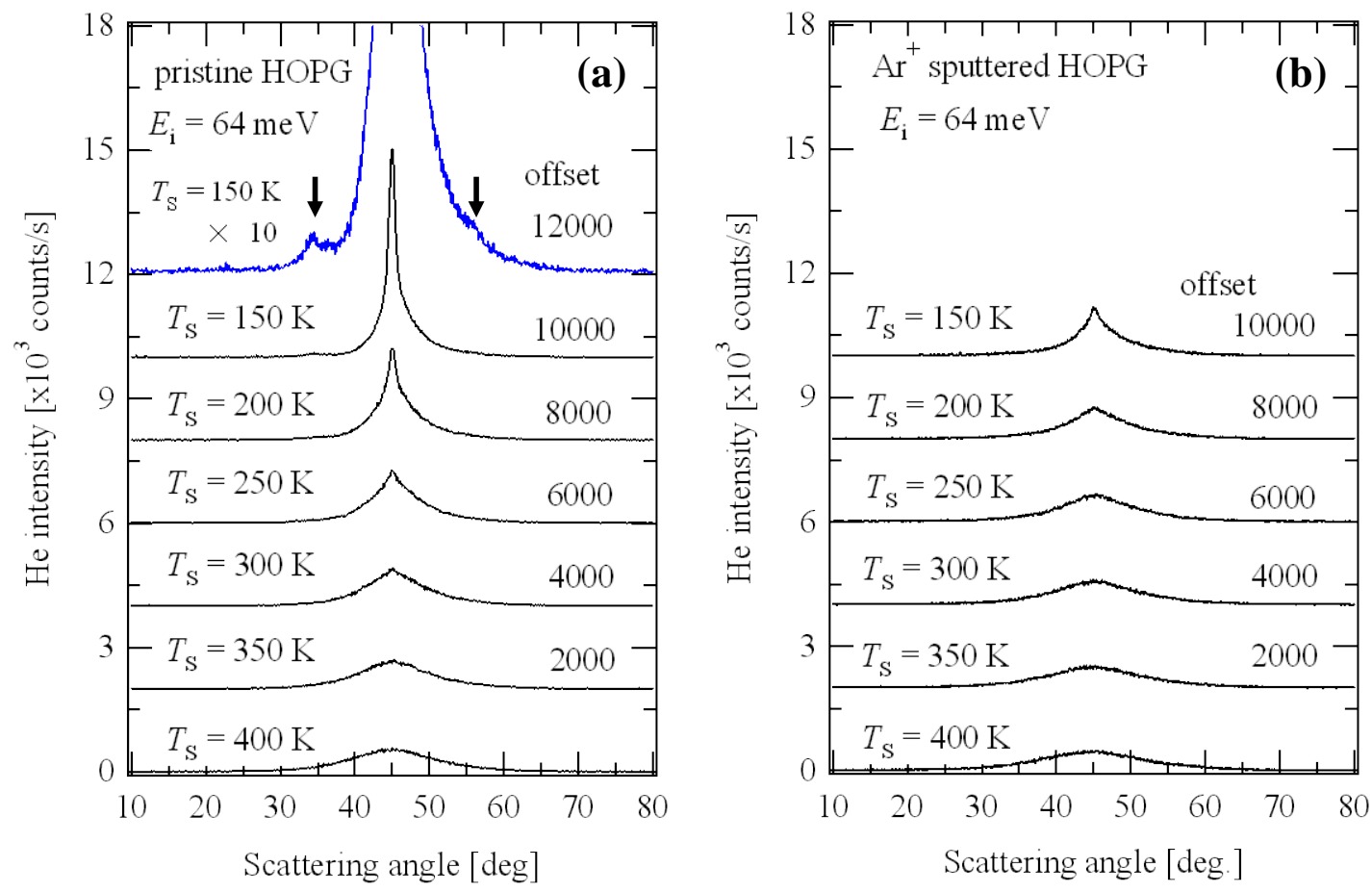
Figure 3

Junepyo Oh, Takahiro Kondo, Daigo Hatake, Yujiro Honma, Keitaro Arakawa, Takahiro Machida and Junji Nakamura
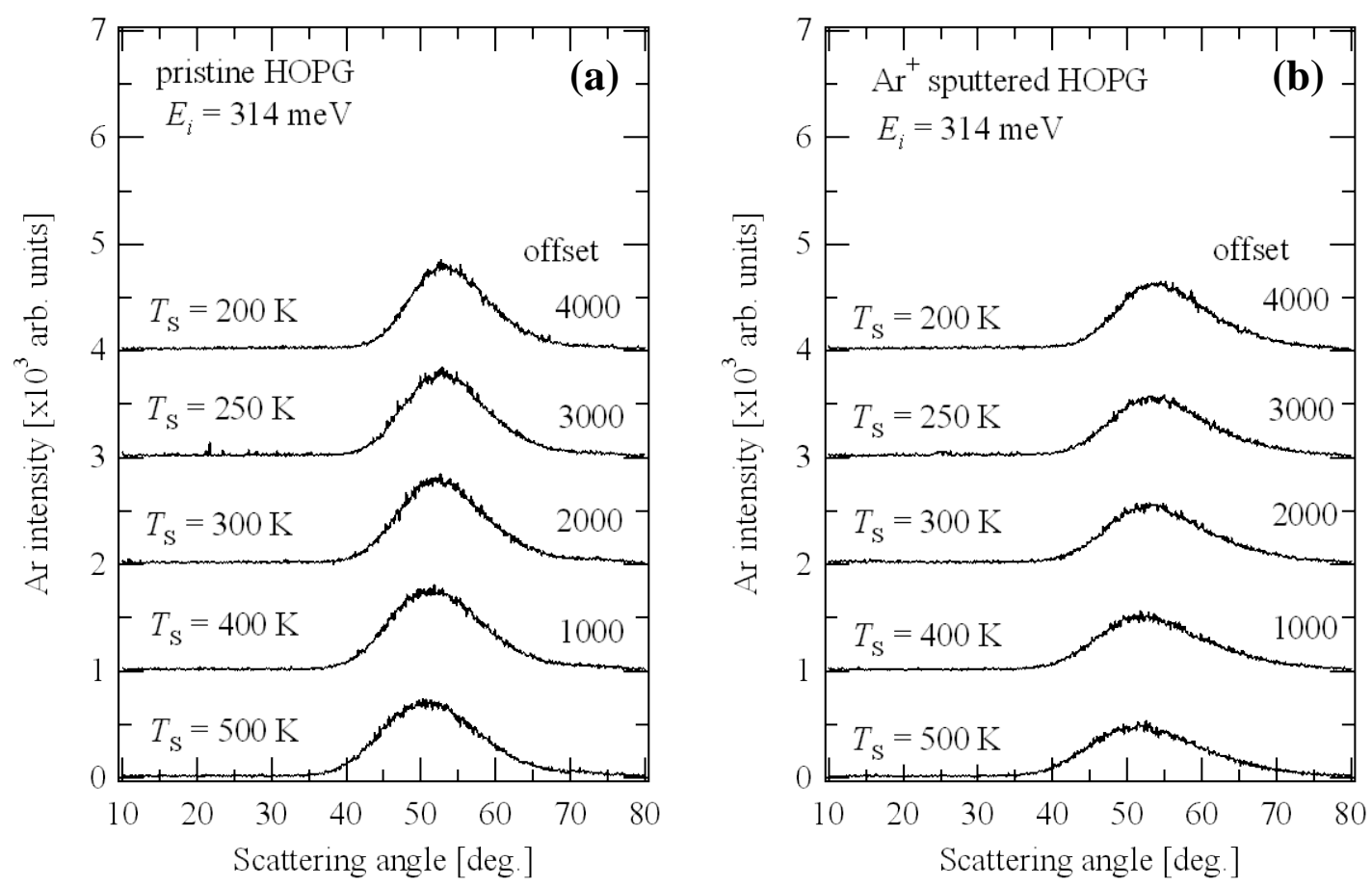
Figure 4

Junepyo Oh, Takahiro Kondo, Daigo Hatake, Yujiro Honma, Keitaro Arakawa, Takahiro Machida and Junji Nakamura

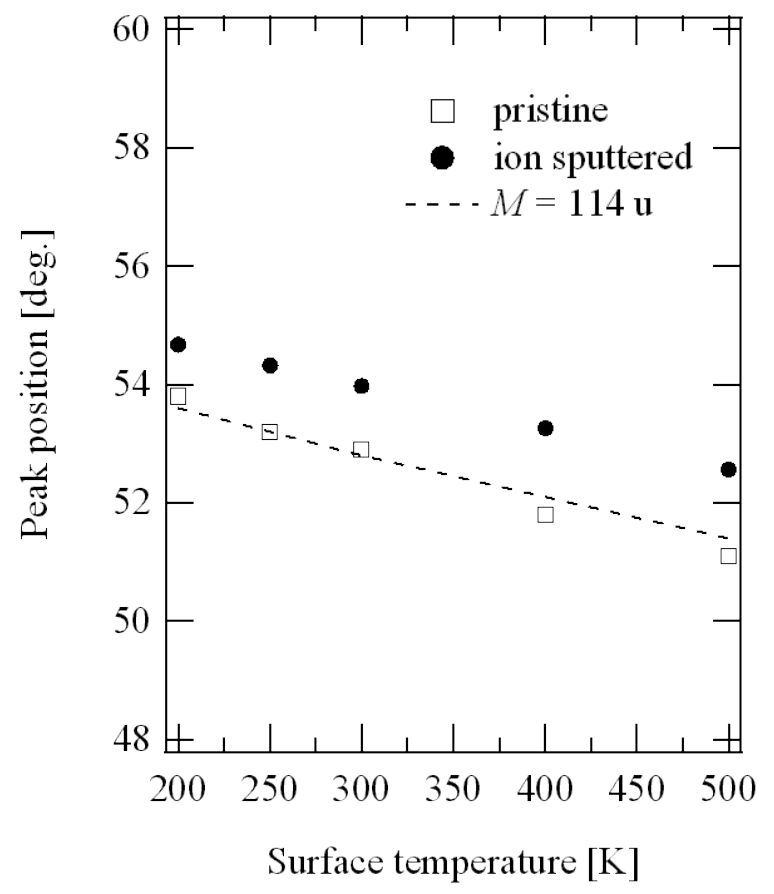


Figure 5

Junepyo Oh, Takahiro Kondo, Daigo Hatake, Yujiro Honma, Keitaro Arakawa, Takahiro Machida and Junji Nakamura

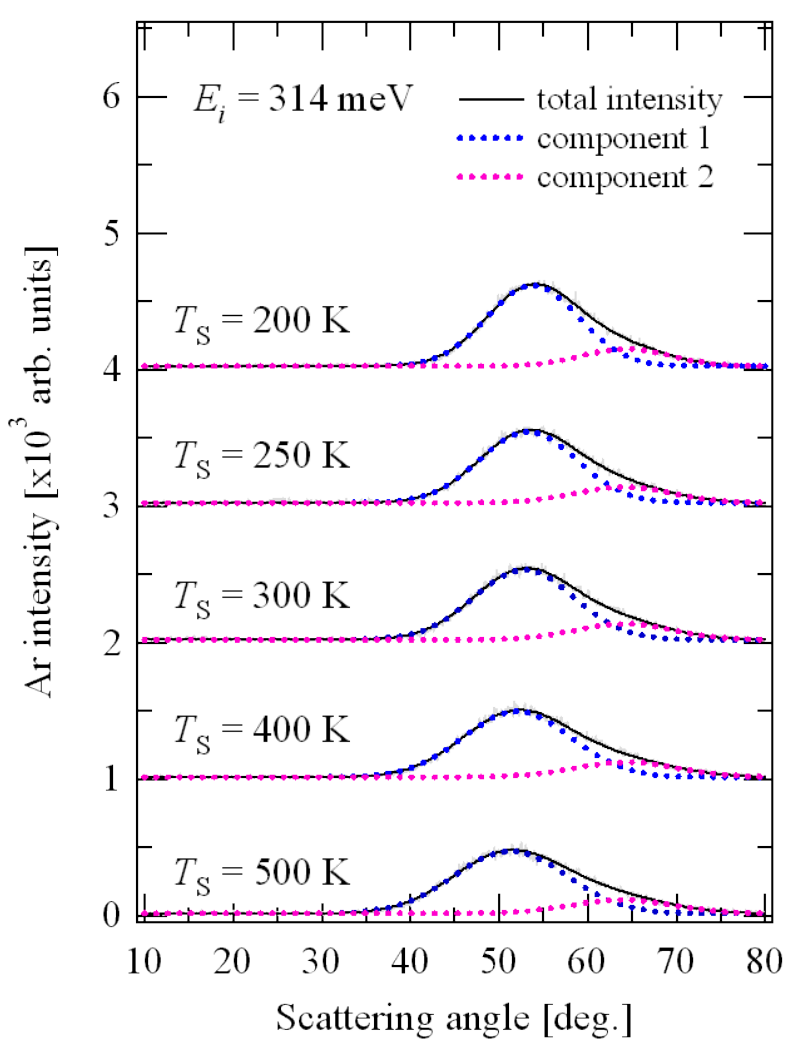

